\title{
The Additive Value of 3D Total Body Imaging for Sequential Monitoring of Skin Lesions: A Case Series
}

\author{
Katarzyna Grochulska a b Brigid Betz-Stablein ${ }^{a, c}$ Chantal Rutjes ${ }^{a}$ \\ Frank Po-Chao Chiu ${ }^{a}$ Scott W. Menzies ${ }^{d}$ H. Peter Soyer ${ }^{a, e}$ Monika Janda $^{f}$ \\ aUniversity of Queensland Diamantina Institute, University of Queensland, Dermatology Research Centre, Brisbane, \\ QLD, Australia; 'bermatology Department, Medical University of Graz, Graz, Austria; 'Cancer and Population \\ Studies, QIMR Berghofer Medical Research Institute, Brisbane, QLD, Australia; dSydney Melanoma Diagnostic \\ Centre, Royal Prince Alfred Hospital and Sydney Medical School, University of Sydney, Sydney, NSW, Australia; \\ eDermatology Department, Princess Alexandra Hospital, Brisbane, QLD, Australia; ${ }^{\mathfrak{f} C e n t r e ~ o f ~ H e a l t h ~ S e r v i c e s ~}$ \\ Research, Faculty of Medicine, University of Queensland, Brisbane, QLD, Australia
}

\section{Keywords}

3D total body imaging $\cdot$ Digital dermoscopy - Early

detection $\cdot$ Melanoma $\cdot$ Long-term monitoring

\begin{abstract}
Background: Timely diagnosis is the cornerstone of melanoma morbidity and mortality reduction. 2D total body photography and dermoscopy are routinely used to assist with early detection of skin malignancies. Polarized 3D total body photography is a novel technique that enables fast image acquisition of almost the entire skin surface. We aimed to determine the added value of 3D total body photography alongside dermoscopy for monitoring cutaneous lesions. Methods: Lesion images from high-risk individuals were assessed for long-term substantial changes via dermoscopy and 3D total body photography. Three case studies are presented demonstrating how 3D total body photography may enhance lesion analysis alongside traditional dermoscopy. Results: 3D total body photography can assist clinicians by presenting cutaneous lesions in their skin ecosystem, thereby providing additional clinical context and enabling a more
\end{abstract}

karger@karger.com www.karger.com/drm

Karger $\stackrel{\text { ' }}{5}$

GOPEN ACCESS
(C) 2021 The Author(s)

Published by S. Karger AG, Basel

This is an Open Access article licensed under the Creative Commons Attribution-NonCommercial-4.0 International License (CC BY-NC) (http://www.karger.com/Services/OpenAccessLicense), applicable to the online version of the article only. Usage and distribution for commercial purposes requires written permission. holistic assessment to aid dermoscopy interpretation. For lesion cases where previous dermoscopy is unavailable, corresponding 3D images can substitute for baseline dermoscopy. Additionally, 3D total body photography is not susceptible to artificial stretch artefacts. Conclusion: 3D total body photography is valuable alongside dermoscopy for monitoring cutaneous lesions. Furthermore, it is capable of surveilling almost the entire skin surface, including areas not traditionally monitored by sequential imaging.

(c) 2021 The Author(s).

Published by S. Karger AG, Basel

\section{Introduction}

Regular skin monitoring is key for early detection of cutaneous malignancies. Multiple strategies are currently employed for short- and long-term lesion monitoring of individually selected melanocytic lesions. Typically, short-term digital dermoscopy monitoring (3-monthly) is performed for suspicious lesions, with the main goal of

This article is part of the Nevi Article Series.
Correspondence to:

Brigid Betz-Stablein, b.betzstablein@uq.edu.au 
Fig. 1. Example of substantial growth. Using total body photography, a pigmented lesion was identified on the right deltoid of a 71-year-old male with a history of primary invasive melanoma. Across 7 months of observation, this lesion demonstrated colour change and an asymmetric increase in size. These changes are clearly distinguishable in both the dermoscopic (a, b) and magnified 3D images (c, d). a Dermoscopy of the lesion reveals classical features of a seborrhoeic keratosis, including comedolike openings (crypts), milia-like cysts, and sharply demarcated borders. b An enlargening hyperkeratotic mass highly suspicious for a squamous cell carcinoma arising within a seborrhoeic keratosis. Histopathological examination revealed an intraepidermal squamous cell carcinoma arising within a seborrhoeic keratosis. Even without dermoscopic images, the magnified 3D image indicates the need of a diagnostic biopsy.
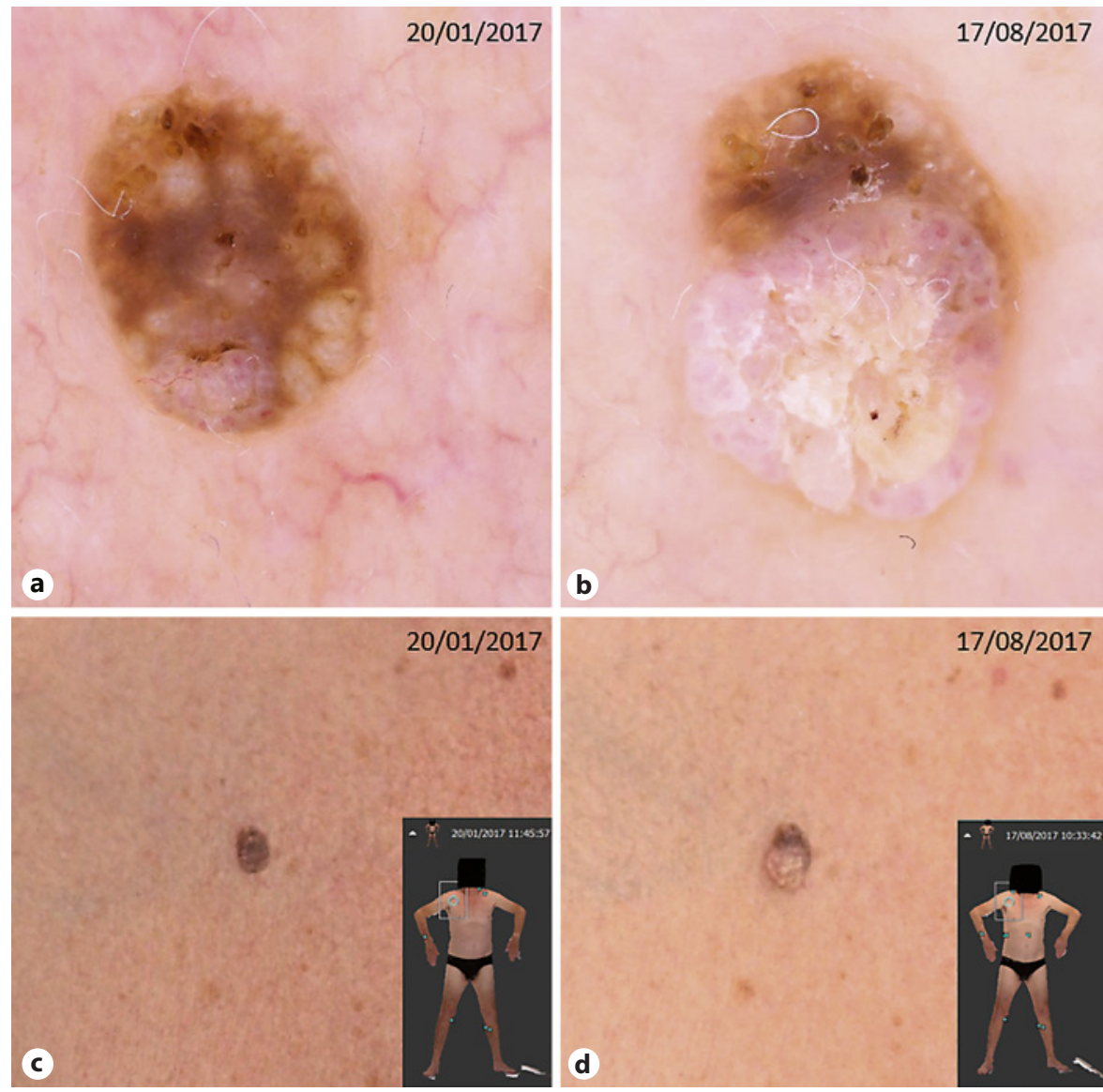

$17 / 08 / 2017$

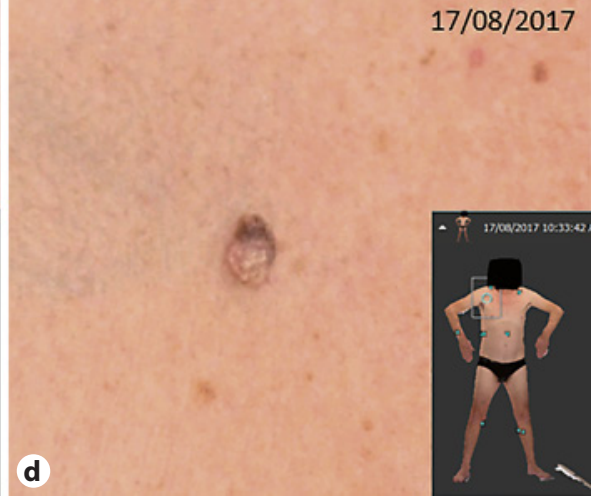

detecting any morphological change that leads to excision. In contrast, long-term dermoscopy follow-up every 6 or 12 months for certain specific types of change is used to detect new or slow-growing melanomas, often in the context of patients with multiple atypical naevi. Both techniques lead to the detection of melanoma, often without specific melanoma dermoscopy features, and both reduce the number of benign lesions excised [1].

Longitudinal dermoscopy follow-up of selected lesions is useful for pre-existing lesions, but is time-consuming and does not usually follow lesions previously regarded as benign or areas of skin without lesions. These inconspicuous areas of skin cannot be neglected because more melanomas develop de novo than arise from a preexisting naevus [2]. Total body photography has been deployed successfully to achieve longitudinal follow-up of the entire skin surface [3], increasing the detection of both melanoma and non-melanoma skin malignancies [4]. Combining 2D total body imaging with digital dermoscopy follow-up has been shown to improve early detection of melanomas while reducing unnecessary exci- sions in high-risk patients [5]. However, this becomes an increasingly time-intensive process as the number of lesions requiring monitoring increases. It has been reported that such surveillance takes $>1 \mathrm{~h}$ for patients with $>100$ naevi in a study setting [6].

$3 \mathrm{D}$ total body photography has recently been introduced, which overcomes some of the limitations of 2D total body photography by streamlining the total body photography process and improving the presentation of curved surfaces. In addition, compared to the acquisition and assessment of multiple $2 \mathrm{D}$ total body images, a single avatar is formed from 92 simultaneously captured images, which are "stitched" together to view almost the entire skin surface. Integrated software provides the ability to assign corresponding dermoscopic images to a lesion of interest. In addition, images taken at follow-up visits (both 3D avatar and dermoscopy) can be compared side by side, facilitating monitoring for change [7]. This paper presents three case studies that illustrate the potential for 3D total body photography to assist clinicians with the assessment of cutaneous lesions. 

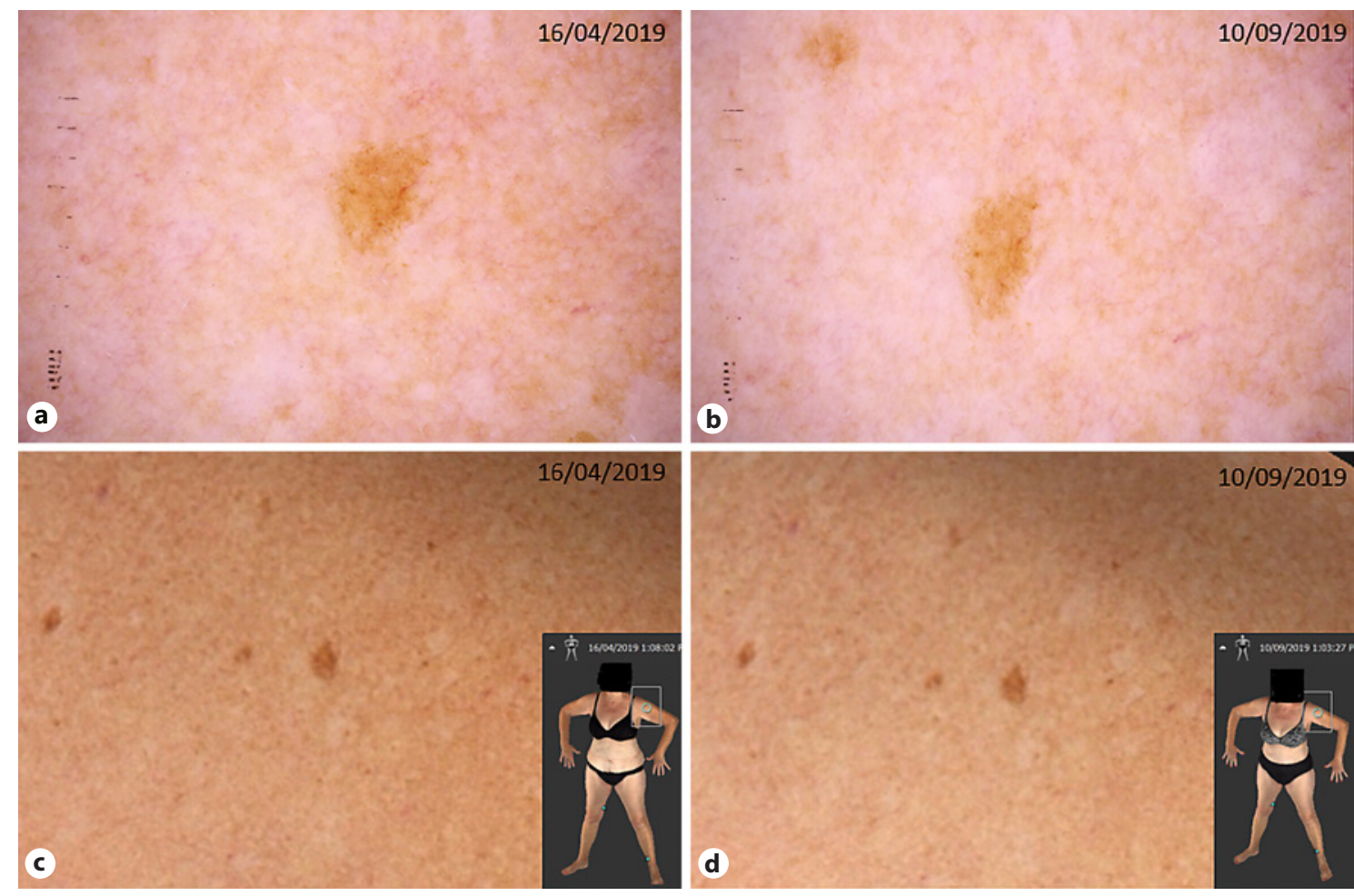

$16 / 04 / 2019$

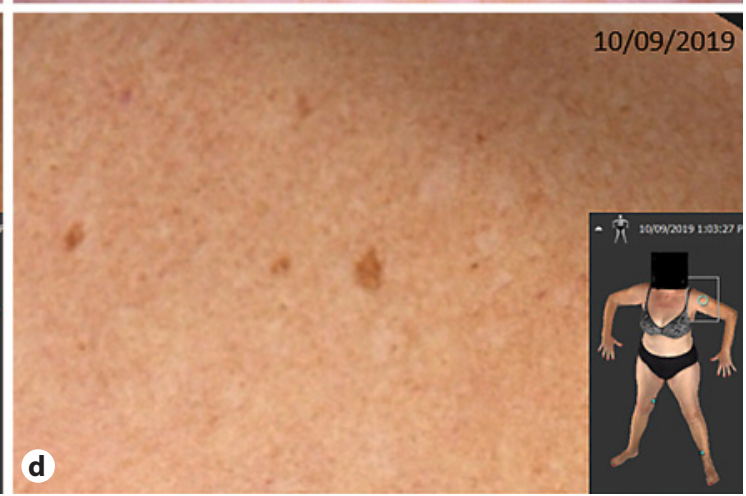

Fig. 2. Example of dermoscopic stretch artefact. The figure shows a benign melanocytic naevus found on the left deltoid of a 67-yearold female with no history of melanoma, which was observed over a period of 5 months. Both dermoscopic images $(\mathbf{a}, \mathbf{b})$ reveal a rather homogenous, tan pigmented lesion with scattered brown globules. The lesion appears to have changed its shape (longer and thinner in $\mathbf{b}$ compared to $\mathbf{a}$ ). In contrast, the high-magnification $3 \mathrm{D}$ total photography images $(\mathbf{c}, \mathbf{d})$ do not demonstrate any change

\section{Methods}

Sample

Examples were selected from the participants taking part in studies with the Dermatology Research Centre between January 2017 and November 2020 from three clinical studies, two following individuals at high risk of melanoma (Changing Naevi, HOPs [8]) and the third following participants from the general population (Mind Your Moles [9]). All participants resided in Queensland, Australia.

\section{Imaging}

Images were obtained using the Vectra ${ }^{\circledR}$ WB360 System for total body captures and VEOS ${ }^{\circledR}$ SLR, LED-flash-based dermoscopy solution with Canon EOS Rebel T6i digital single-lens reflex camera (Canfield Scientific, Inc., Fairfield, NJ, USA). The Vectra ${ }^{\circledR}$ WB360 System uses 92 cameras to simultaneously take standardized polarized images of the whole skin surface. This enables a reconstruction of a $3 \mathrm{D}$ body avatar with integrated software with the ability to assign corresponding dermoscopic images to the lesions of interest. Images taken at follow-up visits can be compared side to side, facilitating monitoring for change [7]. in size and shape. We conclude that the phenomenon in the dermoscopic images represents a stretch artefact due to differences in the pressure applied by the contact plate of the camera while taking the dermoscopic image. Serial 3D photography has the important advantage of visualizing the skin without geometrical errors seen by imaging at different angles using conventional $2 \mathrm{D}$ photography.

\section{Results}

We present three case studies monitoring cutaneous lesions where $3 \mathrm{D}$ total body imaging can replace or assist with the clinical assessment of dermoscopic images (Fig. 1-3).

\section{Discussion}

This case series presents three purposefully selected cases longitudinally monitored using 3D total body photography and digital dermoscopy to highlight the benefits of using both image modalities in parallel.

Assessment of lesions in their clinical context as enabled by $3 \mathrm{D}$ total body photography is notably important in the management of ambiguous lesions [10]. Clinicians not only rely on analytical assessment of indi- 
Fig. 3. De novo lesion. A 48-year-old male with a history of primary melanoma presented with a new pigmented lesion on his lower back (b), which was detected on the $3 \mathrm{D}$ avatar when compared to the previous time point. A dermoscopic image was taken, revealing a reticular naevus, $3 \mathrm{~mm}$ in diameter, with focal central hyperpigmentation and scattered brown globules (a). Upon review of two previously captured total-body 3D images, it became evident that the lesion had not been present 19 months prior (b) and had just been visible as a slightly pigmented spot 12 months prior (c). The naevus was excised, given the relatively quick growth and the patient's history of melanoma, and histopathologically diagnosed as a compound dysplastic naevus. d Without 3D total body photography, it would not have been possible to confirm the de novo appearance of the naevus and to calculate the timeframe of lesion growth.

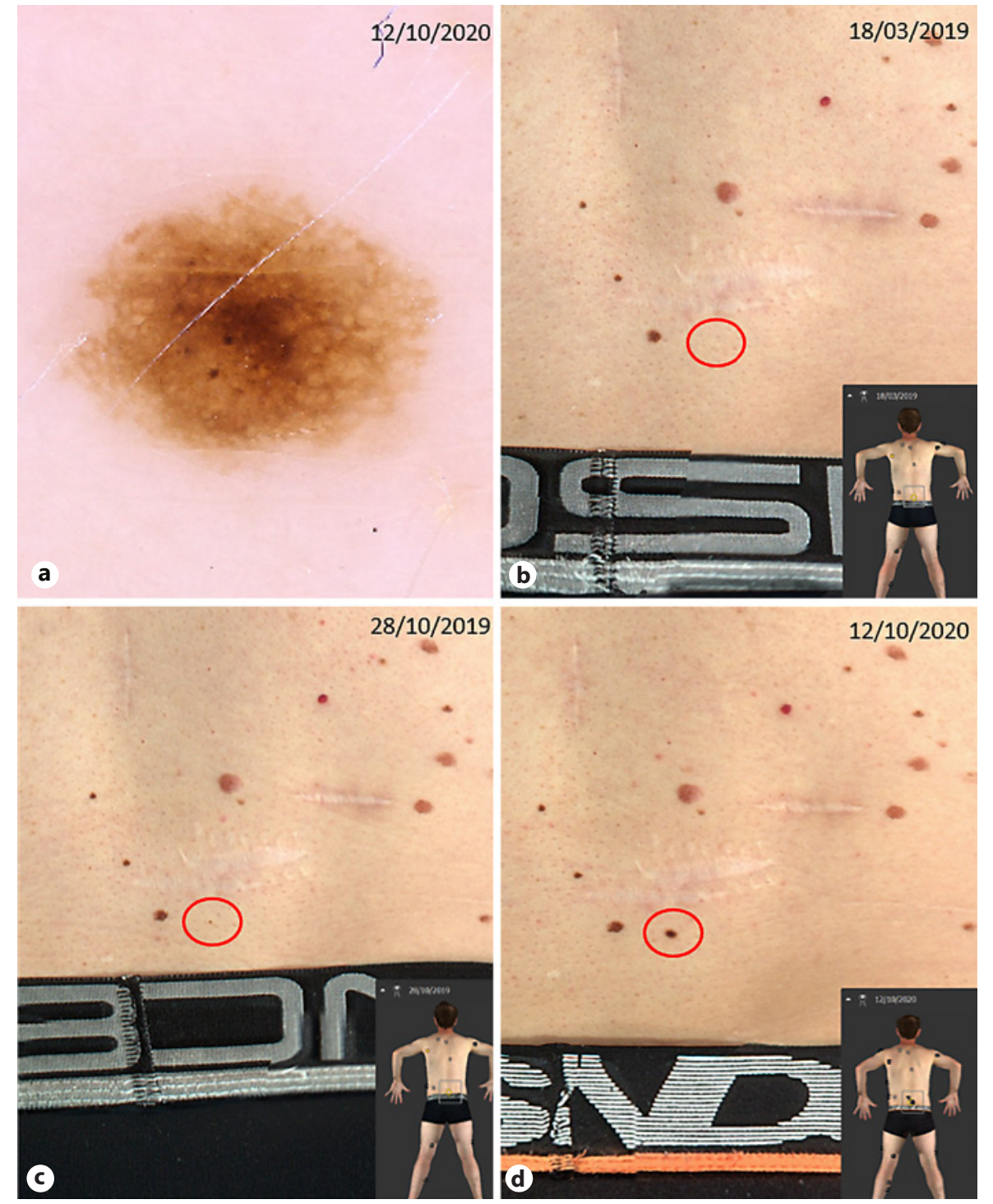

vidual characteristics of pigmented lesions, but more often they evaluate the prevailing pattern of pigmented lesions in an individual and subsequently assess individual lesions relative to their overall skin ecosystem [11]. Although common naevi are all distinct from one another, it has been noticed that the naevi within an individual tend to share common characteristics. Any skin lesions lacking resemblance to the others of an individual stand out (the so-called "ugly duckling") and are more likely to be melanomas [12]. The addition of $3 \mathrm{D}$ imaging is particularly useful for high-risk patients with many naevi where diagnostic biopsy of every suspicious lesion is unfeasible.

Added Value of 3D Total Body Imaging
3D images are particularly useful in telemedicine, providing the physician with additional clinical information and historical context (number, size, and distribution of naevi) as well as other benign non-melanocytic lesions and the degree of photodamage. This additional information provides greater insight into the biologic skin lesion ecosystem of a given person, allowing a more personalized skin cancer screening approach. Previous studies have shown that the accuracy of diagnosis of melanoma is higher when intrapatient comparative approaches are used as opposed to lesion-focused morphological analysis [13]. 3D imaging can be performed remotely, with the assessment of images subsequently conducted by a specialist using teledermatology, thereby improving accessibili- 
ty to patients in rural areas. Additionally, 3D total body imaging provides a promising alternative for the remote diagnosis of lesions by clinicians during pandemics such as COVID-19, where close-up examination may be hindered or unfavorably viewed by patients and doctors alike.

3D imaging may facilitate long-term surveillance as it monitors the entire skin surface rather than individual lesions. Specifically, longitudinal 3D total body photography allows the detection of new de novo skin lesions. Importantly, this may assist in the detection of nodular melanoma at earlier stages, where new screening approaches are needed $[14,15]$. Detection of nodular melanoma is particularly challenging due to the fast growth rate and atypical clinical [16] and dermoscopic [17] presentation, including a higher prevalence of light-coloured/amelanotic lesions and those with symmetry of pigmentation pattern and shape, when compared to other melanoma subtypes. Hence, regular 3D total body imaging with automated recognition of new or changing lesions might be of great help in early detection of nodular melanoma.

Artefacts present in longitudinal dermoscopy imaging can also be compensated for with the addition of 3D total body photography since, as opposed to dermoscopy, no pressure is applied to the skin and common stretch artefacts are minimalized. Furthermore, 3D images are particularly helpful in assessing raised lesions as they can be appreciated from different angles.

The examples presented in this case series were selected for educational purposes and to highlight our experience of incorporating 3D imaging into the clinical setting. The combination of longitudinal $3 \mathrm{D}$ imaging and dermoscopy works as well as, and in certain scenarios better, than $2 \mathrm{D}$ imaging. That being said, at the current resolution, 3D total body imaging does not yet permit evaluation of lesions with the same detail as digital dermoscopy and therefore we recommend the use of 3D total body alongside dermoscopy imaging. As a stand-alone technology, however, it may still offer benefit to detect fast-growing tumours on inconspicuous skin or in association with pre-existing lesions. As technology improves, higher-resolution total body photography will become available. In combination with software-aided matching of images from previous visits, automatic size and angle calibration, as well as artificial intelligence support, it may become the standard of care for clinical monitoring, particularly in high-risk patients, when dermoscopic imaging of every lesion is not feasible.

\section{Conclusion}

3D body imaging is a helpful tool in surveillance of skin lesions, especially in high-risk patients with numerous naevi. The image acquisition is extremely fast and comprehensive. It provides greater clinical context for dermoscopy images of single lesions and may increasingly allow rapid identification of changing or newly arising lesions, even without dermoscopic imaging.

\section{Key Message}

3D total body photography can provide additional information aiding remote melanoma diagnosis, particularly when combined with teledermoscopy.

\section{Acknowledgement}

We thank Joachim Torrano for his help in technical issues.

\section{Statement of Ethics}

The analyzed images were acquired as part of the Changing Naevi Study, Mind Your Moles, and the Higher Outcomes Partnership Study. Ethics approval was obtained from the Human Research Ethics Committees of Metro South Health (HREC/16/ QPAH/37, HREC/16/QPAH/125, HREC/17/QPAH/816) and the University of Queensland HREC (2016000429, 2018000074). All participants provided informed consent.

\section{Conflict of Interest Statement}

H.P. Soyer is a shareholder of e-derm consult $\mathrm{GmbH}$ and MoleMap by Dermatologists Pty Ltd. He regularly provides teledermatological reports for both companies. He also consults for Canfield Scientific, Inc. and is an adviser of First Derm ${ }^{\mathrm{TM}}$.

\section{Funding Sources}

This work was supported by the National Health and Medical Research Council - Centre of Research Excellence scheme (grant No.: APP1099021). H.P. Soyer is also funded by the Medical Research Future Fund - Next Generation Clinical Researcher's Program Practitioner Fellowship (APP1137127). M. Janda is funded by an NHMRC Translating Research into Practice fellowship (APP1151021).
16

Dermatology 2022;238:12-17

DOI: $10.1159 / 000517900$
Grochulska/Betz-Stablein/Rutjes/Chiu/ Menzies/Soyer/Janda 


\section{Author Contributions}

K. Grochulska, C. Rutjes, H.P. Soyer, and F.P.-C. Chiu chose and compared the images. K. Grochulska and B. Betz-Stablein drafted the manuscript. S.W. Menzies and H.P. Soyer participated in the series design. S.W. Menzies, H.P. Soyer, K. Grochulska, and F.P.-C. Chiu provided the interpretation of the results. H.P. Soyer and M. Janda conceptualized the case series. All authors contributed to the writing of the final manuscript.

\section{References}

1 Menzies SW, Chamberlain A, Guitera P, Soyer HP, Cancer Council Australia Melanoma Guidelines Working Party. What is the role of sequential digital dermoscopy imaging in melanoma diagnosis? Sydney: 2018. Available from: https://wiki.cancer.org.au/australia/ Clinical-question:_What-is-the-role-of-sequential-digital-dermoscopy-imaging-inmelanoma-diagnosis\%3F

2 Harley S, Walsh N. A new look at nevus-associated melanomas. Am J Dermatopathol. 1996;18(2):137-41.

3 Kelly JW, Yeatman JM, Regalia C, Mason G, Henham AP. A high incidence of melanoma found in patients with multiple dysplastic naevi by photographic surveillance. Med J Aust. 1997;167(4):191-4

4 Feit NE, Dusza SW, Marghoob AA. Melanomas detected with the aid of total cutaneous photography. Br J Dermatol. 2004;150(4): 706-14.

5 Salerni G, Carrera C, Lovatto L, Puig-Butille JA, Badenas C, Plana E, et al. Benefits of total body photography and digital dermatoscopy ("two-step method of digital follow-up") in the early diagnosis of melanoma in patients at high risk for melanoma. J Am Acad Dermatol. 2012;67(1):e17-27.

6 Haenssle HA, Krueger U, Vente C, Thoms KM, Bertsch HP, Zutt M, et al. Results from an observational trial: digital epilumines- cence microscopy follow-up of atypical nevi increases the sensitivity and the chance of success of conventional dermoscopy in detecting melanoma. J Invest Dermatol. 2006;126(5): 980-5.

7 Rayner JE, Laino AM, Nufer KL, Adams L, Raphael AP, Menzies SW, et al. Clinical perspective of 3D total body photography for early detection and screening of melanoma. Front Med (Lausanne). 2018;5:152.

8 Primiero CA, McInerney-Leo AM, Betz-Stablein B, Whiteman DC, Gordon L, Caffery L, et al. Evaluation of the efficacy of 3D totalbody photography with sequential digital dermoscopy in a high-risk melanoma cohort: protocol for a randomised controlled trial. BMJ Open. 2019;9(11):e032969.

9 Koh U, Janda M, Aitken JF, Duffy DL, Menzies S, Sturm RA, et al. "Mind your Moles" study: protocol of a prospective cohort study of melanocytic naevi. BMJ Open. 2018;8(9): e025857.

10 Carli P, Chiarugi A, De Giorgi V. Examination of lesions (including dermoscopy) without contact with the patient is associated with improper management in about $30 \%$ of equivocal melanomas. Dermatol Surg. 2005; 31(2):169-72.

11 Gachon J, Beaulieu P, Sei JF, Gouvernet J, Claudel JP, Lemaitre M, et al. First prospective study of the recognition process of mela- noma in dermatological practice. Arch Dermatol. 2005;141(4):434-8.

12 Grob J, Bonerandi J. The "ugly duckling" sign identification of the common characteristics of nevi in an individual as a basis for melanoma screening. Arch Dermatol. 1998;134(1): 103-4.

13 Gaudy-Marqueste C, Wazaefi Y, Bruneu Y, Triller R, Thomas L, Pellacani G, et al. Ugly duckling sign as a major factor of efficiency in melanoma detection. JAMA Dermatol. 2017; 153(4):279-84

14 Mar V, Roberts H, Wolfe R, English DR, Kelly JW. Nodular melanoma: a distinct clinical entity and the largest contributor to melanoma deaths in Victoria, Australia. J Am Acad Dermatol. 2013;68(4):568-75.

15 Demierre MF, Chung C, Miller DR, Geller AC. Early detection of thick melanomas in the United States: beware of the nodular subtype. Arch Dermatol. 2005;141(6):745-50.

16 Liu W, Dowling JP, Murray WK, McArthur GA, Thompson JF, Wolfe R, et al. Rate of growth in melanomas: characteristics and associations of rapidly growing melanomas. Arch Dermatol. 2006;142(12): 1551-8.

17 Menzies SW, Moloney FJ, Byth K, Avramidis M, Argenziano G, Zalaudek I, et al. Dermoscopic evaluation of nodular melanoma. JAMA Dermatol. 2013;149(6):699-709. 